\title{
Interaction fluide/structure dans le domaine des moyennes fréquences
}

\author{
Marie de Rochambeau ${ }^{1, a}$, Mohamed Ichchou $^{1}$, Louis Jezequel ${ }^{1}$ et Bernard Troclet ${ }^{2}$ \\ 1 Laboratoire de Tribologie et Dynamique des Systèmes, UMR CNRS 5513, École Centrale de Lyon, LTDS, \\ 36 avenue Guy de Collongue, 69134 Écully Cedex, France \\ 2 Service Vibro-acoustique et dynamique rapide, EADS Astrium ST, 66 route de Verneuil, 78130 Les Mureaux, France
}

Reçu le 30 septembre 2008, accepté le 18 décembre 2008

\begin{abstract}
Résumé - Le présent article traite des interactions fluide/structure dans le domaine des moyennes fréquences. En effet, dans ce domaine fréquentiel, il n'est pas possible de modéliser un système fluide/structure en FEM car la densité modale du milieu fluide est trop importante, ni de le modéliser en SEA car cette modélisation ne serait pas valide. Une méthode hybride FEM/SEA est donc développée : la méthode SEA-like. La méthode SEA-like est une méthode énergétique dont la matrice est calculée avec des méthodes de type «basses fréquences ». Les coefficients SEA-like sont calculés avec une condensation dynamique afin de prendre en compte la densité modale importante dans le système. Dans cet article, la matrice SEA-like d'un système couplé plaque/cavité est calculé avec une analyse modale. On obtient alors une expression simplifiée des coefficients SEA-like. La matrice SEA-like est alors validée dans le domaine des basses fréquences avec modélisation FEM du système couplé et dans le domaine des hautes fréquences avec une modélisation SEA du système couplé.
\end{abstract}

Mots clés : Interaction fluide / structure / analyse modale / analyse par éléments-finis / SEA / SEA-like

\begin{abstract}
Fluid-structure interaction in the mid-frequency domain. The modelling of the fluidstructure interaction in the mid-high frequency domain is problematic. Indeed, the modal density of the fluid media does not allow the modelling of the fluid-structure system with FEM or modal analysis because the calculation would not be efficient. The SEA cannot be used either because this method is not precise in the Mid-Frequency domain. A hybrid FEM-SEA method is therefore developed: the SEA-like method. The SEA-like method is an energetic method which coefficients are calculated with low-frequency methods. The high modal density of the fluid-structure coupled system is treated using sub-structuration in the calculation of the SEA-like coefficients. Here, a modal analysis is used to calculate the SEA-like matrix of a panel-cavity coupled system. A simplified expression of the SEA-like coefficients is therefore obtained. The SEA-like matrix of the coupled system is then validated in both low- and high-frequency domains using respectively FEM and SEA models.
\end{abstract}

Key words: Fluide-structure interachion / modal analysis / finite-element analysis / SEA / SEA-like

\section{Introduction}

Dans le domaine de l'aéronautique, les excitations des structures sont aléatoires et large-bande. Les structures doivent alors être modélisées sur une large bande fréquentielle. Or les méthodes utilisées varient suivant la densité modale des systèmes modélisés : dans le domaine des basses fréquences, les méthodes de modélisation utilisées sont la Méthode des Éléments-Finis (FEM) ou

\footnotetext{
a Auteur pour correspondance :

Marie.de-Rochambeau@ec-lyon.fr
}

l'Analyse Modale; en hautes fréquences, compte tenu du facteur de recouvrement modal élevé, des méthodes énergétiques sont préférées, comme la SEA (Statistical Energetic Analysis). Cependant, il existe une zone entre ces deux domaines fréquentiels où aucune de ces méthodes n'est utilisable de façon satisfaisante : le facteur de recouvrement modal est trop faible pour utiliser la SEA de manière fiable et est trop élevé pour utiliser la FEM de manière efficace. Il s'agit du domaine des moyennes fréquences. Dans ce domaine, une méthode énergétique et hybride FEM-SEA est alors développée : la méthode 


\section{Nomenclature}

\begin{tabular}{|c|c|}
\hline$A_{\mathrm{s}}$ & surface de la plaque \\
\hline$a$ & largeur de la plaque \\
\hline$b$ & longueur de la plaque \\
\hline$c$ & profondeur de la cavité \\
\hline$c_{0}$ & célérité du son dans l'air \\
\hline$D$ & rigidité en flexion de la plaque \\
\hline$E_{\mathrm{a}}$ & énergie vibratoire de la structure \\
\hline$E_{\mathrm{s}}$ & énergie acoustique de la cavité \\
\hline$h_{\mathrm{s}}$ & épaisseur de la plaque \\
\hline$L$ & opérateur linéaire de la plaque \\
\hline$p$ & champ de pression dans la cavité \\
\hline$p_{\mathrm{e}}$ & champ de pression extérieur excitant la plaque \\
\hline$q_{\text {ext }}$ & champ des sources acoustiques dans la cavité \\
\hline$\Re(x)$ & partie réelle du nombre complexe $x$ \\
\hline$v$ & champ de vitesse dans la plaque \\
\hline$\Phi_{n}^{\mathrm{a}}$ & $n^{\text {ième }}$ mode de cavité rigide \\
\hline$\Phi_{m}^{\mathrm{s}}$ & $m^{\text {ième }}$ mode de plaque simplement appuyée \\
\hline$\eta_{\mathrm{an}}$ & amortissement du $n^{\text {ième }}$ mode de cavité rigide \\
\hline$\eta_{\mathrm{s} m}$ & amortissement $\mathrm{du} \mathrm{m}^{\text {ième }}$ mode de plaque \\
\hline$v_{\mathrm{s}}$ & coefficient de Poisson de la plaque \\
\hline$\rho_{0}$ & masse volumique de l'air \\
\hline$\rho_{\mathrm{s}}$ & masse volumique de la plaque \\
\hline$\omega$ & pulsation \\
\hline $\mathrm{a}$ & indice ou exposant faisant référence à la cavité \\
\hline $\mathrm{s}$ & indice ou exposant faisant référence à la plaque \\
\hline
\end{tabular}

SEA-like. Cette dernière méthode est une méthode énergétique moins restrictive que la méthode SEA et dont les coefficients sont calculés par des méthodes basses fréquences.

La méthode SEA-like a été étudiée essentiellement sur des systèmes présentant une interaction structure/structure (voir Mace [1,2] et Hiverniau [3]). Mace s'est attaché à écrire une formule applicable à n'importe quel système qui permet de calculer les coefficients de la matrice SEA-like et qui est basée sur une analyse modale du système complet.

Cependant, lorsque un système présentant une interaction fluide/structure doit être modélisé avec la méthode SEA-like, il devient judicieux d'effectuer une condensation dynamique pour simplifier l'analyse modale du système car la densité modale du système fluide peut être très importante. Pan et al. (voir [4-6]) entre autres, se sont attachés à décrire un système composé d'une plaque couplée avec une cavité en utilisant les modes de chacun des sous-systèmes découplés.

Ici, les coefficients SEA-like d'un système couplé plaque/cavité sont calculés avec une méthode de condensation dynamique, puis sont validés dans les domaines des basses fréquences et des hautes fréquences avec des modélisations FEM et SEA du système étudié.

\section{Modélisation}

\subsection{Description du système et de sa matrice SEA-like}

Le système étudié ici est une plaque de dimensions $(a \times b)$ couplée avec une cavité rectangulaire de dimensions $(a \times b \times c)$. Le champ vibratoire dans la plaque est décrit par la vitesse normale de la plaque $v(x, y, \omega)$, et celui de la cavité est décrit par le champ de pression.

Le système est décomposé en deux sous-systèmes : le sous-système plaque et le sous-système cavité. La matrice SEA-like du système est calculée avec les énergies vibratoires ainsi que les puissances injectées moyennées suivant la fréquence dans chaque sous-système :

$$
\left(\begin{array}{c}
\left\langle E_{\mathrm{s}}\right\rangle \\
\left\langle E_{\mathrm{a}}\right\rangle
\end{array}\right)=\left(\begin{array}{cc}
A_{\mathrm{ss}} & A_{\mathrm{sa}} \\
A_{\mathrm{as}} & A_{\mathrm{aa}}
\end{array}\right)\left(\begin{array}{c}
\left\langle P_{\mathrm{inj}, \mathrm{s}}\right\rangle \\
\left\langle P_{\mathrm{inj}, \mathrm{a}}\right\rangle
\end{array}\right)
$$

Deux cas d'excitation sont considérés pour le calcul des coefficients SEA-like : le premier cas d'excitation est l'excitation de la plaque seule, qui permet de calculer les coefficients $A_{\mathrm{ss}}$ et $A_{\mathrm{as}}$, en considérant, dans l'équation (1) que $\left\langle P_{\text {inj,a }}\right\rangle=0$; le deuxième cas d'excitation est l'excitation de la cavité seule, qui permet de calculer les coefficients $A_{\mathrm{sa}}$ and $A_{\mathrm{aa}}$ en considérant dans l'équation (1) que $\left\langle P_{\mathrm{inj}, \mathrm{s}}\right\rangle=0$. Ce cas d'excitation ne sera pas considéré ici.

\subsection{Calcul de $\boldsymbol{A}_{s s}$}

\subsection{1 Équation matricielle du système}

Le système est décrit par une analyse modale par condensation dynamique. Les équations du système sont donc projetées sur les modes des systèmes découplés : les modes décrivant la cavité sont les modes rigides :

$$
\Phi_{n}^{\mathrm{a}}(x, y, z)=\cos \left(\frac{m \pi x}{a}\right) \cos \left(\frac{n \pi y}{b}\right) \cos \left(\frac{t \pi z}{c}\right)
$$

de fréquence propre :

$$
f_{\mathrm{a} n}=\frac{\omega_{\mathrm{a} n}}{2 \pi}=\frac{c_{0}}{2 \pi} \sqrt{\left(\frac{m \pi}{a}\right)^{2}+\left(\frac{n \pi}{b}\right)^{2}+\left(\frac{t \pi}{c}\right)^{2}}
$$

et les modes décrivant la plaque sont les modes de plaque simplement appuyée :

$$
\Phi_{m}^{\mathrm{s}}(x, y)=\sin \left(\frac{p \pi x}{a}\right) \sin \left(\frac{q \pi y}{b}\right)
$$

de fréquence propre :

$$
f_{\mathrm{s} m}=\frac{\omega_{\mathrm{s} m}}{2 \pi}=\frac{1}{2 \pi} \sqrt{\frac{D}{\rho_{\mathrm{s}} h_{\mathrm{s}}}}\left(\left(\frac{p \pi}{a}\right)^{2}+\left(\frac{q \pi}{b}\right)^{2}\right)
$$

On considère que la plaque seule est excitée par un champ de pression $p_{\mathrm{e}}(x, y, \omega)$. La vitesse normale dans la plaque vérifie alors l'équation suivante :

$$
\begin{aligned}
& L v(x, y, z, \omega)-\rho_{\mathrm{s}} h_{\mathrm{s}} \omega^{2} v(x, y, z, \omega)= \\
& j \omega\left(p(x, y, z=1, \omega)-p_{\mathrm{ext}}(x, y, \omega)\right)
\end{aligned}
$$


Si on prend en compte le caractère orthogonal des modes et que l'on introduit l'expression modale du champ de pression dans la cavité, l'équation (6) devient :

$$
\begin{aligned}
& \frac{j M_{m}^{\mathrm{s}}}{A_{\mathrm{s}} \rho_{0} c_{0} \omega}\left(\omega_{\mathrm{s} m}^{2}+j \eta_{\mathrm{s} m} \omega_{\mathrm{s} m}^{2}-\omega^{2}\right) V_{m} \\
& \quad+\frac{1}{\rho_{0} c_{0}} \sum_{n} B_{n, m} P_{n}=\frac{1}{A_{\mathrm{s}} \rho_{0} c_{0}} \int_{A_{\mathrm{s}}} p_{\mathrm{ext}} \Phi_{m}^{\mathrm{s}} \mathrm{d} s
\end{aligned}
$$

où $M_{m}^{\mathrm{s}}=\rho_{\mathrm{s}} h_{\mathrm{s}} \int_{A_{\mathrm{s}}} \Phi_{m}^{\mathrm{s}} \mathrm{d} s$ est la masse modale de la plaque.

Le couplage spatial entre la plaque et la cavité est exprimé dans la matrice de couplage $B$ :

$$
B_{n, m}=\frac{1}{A_{\mathrm{s}}} \int_{A_{\mathrm{s}}} \Phi_{m}^{\mathrm{s}} \Phi_{n}^{\mathrm{a}} \mathrm{d} s
$$

La cavité est décrite par l'équation de Helmholtz :

$$
\Delta p+\frac{\omega^{2}}{c_{0}^{2}} p=0
$$

Sur les parois rigides, le champ de pression $p$ vérifie la condition d'imperméabilité :

$$
\frac{\partial p}{\partial n}=0
$$

Sur la plaque, $p$ vérifie la condition de continuité de vitesses :

$$
\frac{\partial p}{\partial n}=-j \rho_{0} \omega w
$$

Le théorème de Green est utilisé sur l'équation (9) et la pression dans la cavité et la vitesse normale de la plaque sont projetées sur les modes découplés. On obtient alors l'équation modale correspondant au $n^{\text {ième }}$ mode de cavité :

$$
\begin{aligned}
\frac{j M_{n}^{\mathrm{a}}}{\omega \rho_{0} c_{0} A_{\mathrm{s}}}\left(\omega_{\mathrm{a} n}^{2}+j \eta_{\mathrm{a} n} \omega_{\mathrm{a} n}^{2}\right. & \left.-\omega^{2}\right) P_{n} \\
& -\rho_{0} c_{0} \sum_{m} B_{n, m} V_{m}=0
\end{aligned}
$$

où $M_{n}^{\mathrm{a}}$ est la masse modale de la cavité.

Les équations (12) et (7) permettent finalement d'écrire l'équation matricielle du système couplé :

$$
\left(\begin{array}{cc}
\chi_{\mathrm{s}} & \frac{1}{\rho_{0} c_{0}}{ }^{t} B \\
-\rho_{0} c_{0} B & \chi_{\mathrm{a}}
\end{array}\right)\left(\begin{array}{c}
V \\
P
\end{array}\right)=\left(\begin{array}{c}
\chi_{\mathrm{s}} Q_{\mathrm{s}} \\
0
\end{array}\right)
$$

$\chi_{\mathrm{s}}$ est la matrice de transfert de la plaque découplée, $\chi_{\mathrm{a}}$, la matrice de transfert de la cavité découplée et $Q_{\mathrm{s}}$ est le vecteur des forces généralisées.

\subsubsection{Calcul de $A_{\text {ss }}$}

Dans le cas où seule la plaque est excitée, le coefficient de la matrice SEA-like $A_{\mathrm{sS}}$ peut être calculé avec l'équation (1) :

$$
A_{\mathrm{ss}}=\frac{\left\langle E_{\mathrm{s}, \mathrm{s}}\right\rangle}{\left\langle P_{\mathrm{inj}, \mathrm{s}}\right\rangle}
$$

Dans le domaine des hautes fréquences, il est courant d'assimiler l'énergie potentielle de la plaque à son énergie cinétique lors de l'utilisation de méthodes énergétiques comme la SEA (voir [7]). Ici, cette approximation n'est pas considérée. L'énergie de la plaque est donc calculée à partir de son énergie cinétique et de son énergie potentielle de déformation. Ainsi, si l'on exprime la vitesse normale dans la plaque en fonction de sa projection modale, l'énergie de la plaque moyennée fréquentiellement quand la plaque est excitée devient :

$$
\begin{aligned}
\left\langle E_{\mathrm{s}, \mathrm{s}}\right\rangle=\frac{A_{\mathrm{s}}}{16} \frac{1}{\Delta \omega} & \int_{\Delta \omega} \sum_{m}\left|V_{m}\right|^{2} \\
& \times\left(\rho_{\mathrm{s}} h_{\mathrm{s}}+\frac{D \pi^{4}}{\omega^{2}}\left(\frac{p_{m}^{2}}{a^{2}}+\frac{q_{m}^{2}}{b^{2}}\right)^{2}\right) \mathrm{d} \omega
\end{aligned}
$$

Pour calculer le vecteur des vitesses quadratiques $\left|V_{m}\right|^{2}$, il est nécessaire de faire une hypothèse sur l'excitation de la plaque : on suppose que celle-ci est de type « rain-on-the-roof », c'est-à-dire qu'elle est spatialement et fréquentiellement décorrelée. Le champ de pression $p_{\mathrm{e}}$ vérifie alors l'hypothèse suivante :

$$
\begin{aligned}
& \iint_{A_{\mathrm{s}}} p_{\text {ext }}^{*}(x, y ; \omega) \Phi_{p}^{\mathrm{s}}(x, y) p_{\text {ext }}^{*}\left(x^{\prime}, y^{\prime} ; \omega\right) \\
& \quad \times \Phi_{q}^{\mathrm{s}}\left(x^{\prime}, y^{\prime}\right) \mathrm{d} x \mathrm{~d} y \mathrm{~d} x^{\prime} \mathrm{d} y^{\prime}=S_{p_{\text {ext }}}(\omega) j_{p q}^{\mathrm{s}}(\omega)
\end{aligned}
$$

avec :

$$
j_{p q}^{\mathrm{s}}(\omega)=\delta_{p, q} \int_{A_{\mathrm{s}}} h_{\mathrm{s}} \rho_{\mathrm{s}}\left(\Phi_{p}^{\mathrm{s}}(x, y)\right)^{2} \mathrm{~d} x \mathrm{~d} y
$$

La fonction $j_{p q}^{\mathrm{s}}$ est appelée « joint acceptance » et décrit la corrélation fréquentielle de l'excitation. Ici, elle ne dépend pas de la fréquence. L'excitation est donc décorrelée en fréquence.

On obtient ainsi l'expression de l'énergie dans la cavité moyennée sur les fréquences :

$$
\begin{aligned}
\left\langle E_{\mathrm{s}, \mathrm{s}}\right\rangle=\frac{1}{32} \frac{\rho_{\mathrm{s}} h_{\mathrm{s}}}{\rho_{0}^{2} c_{0}^{2}} & \frac{1}{\Delta \omega} \int_{\Delta \omega} S_{p_{\mathrm{ext}}} \sum_{m, p} \frac{\left|\left(H_{\mathrm{ss}}\right)_{m, p}\right|^{2}}{\left|\chi_{\mathrm{s}, p}\right|^{2}} \\
& \times\left(\rho_{\mathrm{s}} h_{\mathrm{s}}+\frac{D \pi^{4}}{\omega^{2}}\left(\frac{p_{m}^{2}}{a^{2}}+\frac{q_{m}^{2}}{b^{2}}\right)\right) \mathrm{d} \omega
\end{aligned}
$$

La puissance injectée dans la plaque s'exprime de la façon suivante :

$$
P_{\mathrm{inj}, \mathrm{s}}=\frac{1}{2} \Re\left(\int_{A_{\mathrm{s}}} p_{\text {ext }}^{*}(x, y ; \omega) v(x, y ; \omega) \mathrm{d} x \mathrm{~d} y\right)
$$

L'hypothèse sur l'excitation de la plaque conduit à l'expression de la puissance injectée moyennée :

$$
\left\langle P_{\mathrm{inj}, \mathrm{s}}\right\rangle=\frac{1}{8} \frac{\rho_{\mathrm{s}} h_{\mathrm{s}}}{\rho_{0} c_{0}} \frac{1}{\Delta \omega} \int_{\Delta \omega} \Re\left(S_{p_{\mathrm{ext}}}(\omega) \sum_{m} \frac{H_{\mathrm{ss}(m, m)}}{\chi_{\mathrm{s}, m}}\right) \mathrm{d} \omega
$$


En utilisant les équations (14), (18) et (20), on obtient le premier coefficient de la matrice SEA-like :

$$
\begin{aligned}
& A_{\mathrm{ss}}=\frac{1}{4 \rho_{0} c_{0}} \\
& \times \frac{\int_{\Delta \omega} \sum_{m, p} \frac{\left|\left(H_{\mathrm{ss}}\right)_{m, p}\right|^{2}}{\left|\chi_{\mathrm{s}, p}\right|^{2}}\left(\rho_{\mathrm{s}} h_{\mathrm{s}}+\frac{D \pi^{4}}{\omega^{2}}\left(\frac{p_{m}^{2}}{a^{2}}+\frac{q_{m}^{2}}{b^{2}}\right)^{2}\right) \mathrm{d} \omega}{\int_{\Delta \omega} \Re\left(\sum_{m} \frac{H_{\mathrm{ss}(m, m)}}{\chi_{\mathrm{s}, \mathrm{m}}}\right) \mathrm{d} \omega}
\end{aligned}
$$

\subsection{Calcul de $\boldsymbol{A}_{\text {as }}$}

Le coefficient $A_{\text {as }}$ est calculé de la même manière que le coefficient $A_{\mathrm{ss}}$. D'après l'équation (1), on peut écrire que :

$$
A_{\mathrm{as}}=\frac{\left\langle E_{\mathrm{a}, \mathrm{s}}\right\rangle}{\left\langle P_{\mathrm{inj}, \mathrm{s}}\right\rangle}
$$

L'énergie vibratoire dans la cavité est la somme de son énergie cinétique et de son énergie potentielle de déformation. L'énergie vibratoire dans la cavité moyennée selon la fréquence s'exprime alors de la façon suivante :

$$
\begin{aligned}
& \left\langle E_{\mathrm{a}, \mathrm{s}}\right\rangle=\frac{\rho_{\mathrm{s}} h_{\mathrm{s}}}{64} \frac{V}{A_{\mathrm{s}}} \frac{1}{\Delta \omega} \int_{\Delta \omega} S_{p_{\mathrm{ext}}} \\
& \times \sum_{n, p} \frac{\left|\left(H_{\mathrm{as}}\right)_{n, p}\right|^{2}}{\left|\chi_{\mathrm{s}, p}\right|^{2}}\left(\frac{\pi^{2}}{\rho_{0} \omega^{2}}\left(\frac{m_{n}^{2}}{a^{2}}+\frac{n_{n}^{2}}{b^{2}}+\frac{t_{n}^{2}}{c^{2}}\right)+\frac{1}{\rho_{0} c_{0}^{2}}\right) \mathrm{d} \omega
\end{aligned}
$$

L'expression de la puissance injectée dans la plaque (Éq. (20)) permet finalement de donner l'expression du coefficient $A_{\text {as }}$ :

$$
\begin{aligned}
& A_{\mathrm{as}}=\frac{1}{8 \rho_{0} c_{0}} \frac{V}{A_{\mathrm{s}}} \\
& \times \frac{\int_{\Delta \omega} \sum_{n, p} \frac{\left|\left(H_{\mathrm{as}}\right)_{n, p}\right|^{2}}{\left|\chi_{\mathrm{s}, p}\right|^{2}}\left(\frac{\pi^{2}}{\rho_{0} \omega^{2}}\left(\frac{m_{n}^{2}}{a^{2}}+\frac{n_{n}^{2}}{b^{2}}+\frac{t_{n}^{2}}{c^{2}}\right)+\frac{1}{\rho_{0} c_{0}^{2}}\right) \mathrm{d} \omega}{\int_{\Delta \omega \Re}\left(\sum_{m} \frac{H_{\mathrm{ss}(m, m)}}{\chi_{\mathrm{s}, m}}\right) \mathrm{d} \omega}
\end{aligned}
$$

\section{Résultats}

Le système modélisé est constitué d'une plaque de dimensions $0,868 \mathrm{~m} \times 1,15 \mathrm{~m}$ et de $5,8 \times 10^{-3} \mathrm{~m}$ d'épaisseur couplée à une cavité de $1 \mathrm{~m}$ de profondeur. La matrice SEA-like de ce système est calculée puis validée en basses fréquences et hautes fréquences par des modélisations FEM et SEA du système couplé.

\subsection{Validation dans le domaine des basses fréquences}

La modélisation basses fréquences est effectuée par comparaison avec un modèle FEM du système couplé. Le système est excité par une source ponctuelle sur la plaque, puis les énergies dans les deux sous-systèmes sont calculées avec les méthodes FEM et SEA-like et comparées (voir Fig. 1).

La différence que l'on observe entre les deux méthodes est due principalement à deux facteurs. Le premier est que la méthode SEA-like étant une méthode énergétique moyennée dans l'espace et le temps, elle donne des résultats approchés, bien que satisfaisants sur la bande de fréquence considérée : on remarque que les écarts entre les énergies calculées par les deux méthodes sont minimes.

Le deuxième facteur provient de la différence entre la Méthode Éléments-Finis et la méthode modale utilisée pour calculer les coefficients SEA-like : dans cette dernière méthode, les modes utilisés pour décrire le champ de pression dans la cavité sont des modes de cavité rigide. Il n'est alors pas possible de décrire le champ de pression correspondant à une plaque flexible dans la cavité : le couplage entre la plaque et la cavité est modifié, ce qui se répercute sur les énergies dans les deux sous-systèmes.

Pour compléter la base modale décrivant la cavité, il faudrait rajouter des modes supplémentaires donnés par le champ de pression lorsque le champ de vitesse de la plaque correspond à un de ses modes. Ces derniers modes de cavité permettent de prendre en compte le couplage entre les deux sous-systèmes et permettrait d'approcher le résultat de manière plus satisfaisante.

\subsection{Validation dans le domaine des hautes fréquences}

Les Coefficients d'Influence Énergétiques (CIE) du système couplé sont calculés par les deux méthodes (Fig. 2). Le calcul SEA est effectué à partir de la théorie de Maidanik (voir [8]).

Les domaines des basses fréquences, moyennes fréquences et hautes fréquences sont définis par le recouvrement modal dans la cavité (voir [6]).

Dans le domaine des basses fréquences, la courbe des CIE calculés par la méthode SEA-like présente de fortes oscillations, dues au faible recouvrement modal dans la cavité. La SEA, qui n'est qu'approximative dans cette zone de fréquences, ne présente pas ces oscillations. Dans le domaine des moyennes fréquences, au-delà de $800 \mathrm{~Hz}$, le facteur de recouvrement modal augmente dans la cavité et la courbe SEA-like est plus lisse et converge vers la courbe SEA. Dans le domaine des hautes fréquences (audessus de $2000 \mathrm{~Hz}$ ), la courbe SEA présente un pic qui est le pic de rayonnement de la plaque dans la cavité. La courbe SEA-like étant calculée de manière discrète en fréquence, continue sa progression. 

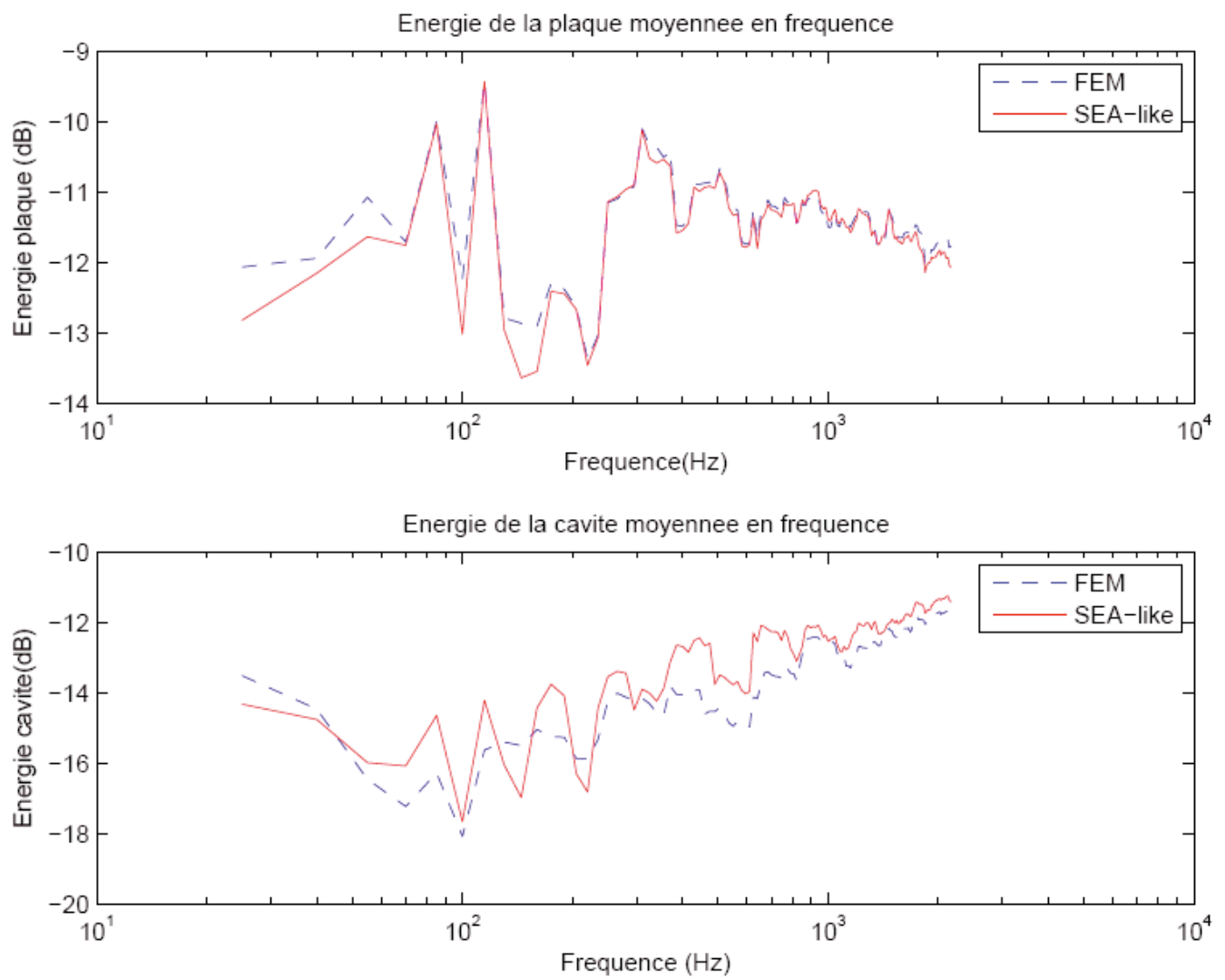

Fig. 1. Énergies dans les deux sous-systèmes moyennées sur les fréquences.
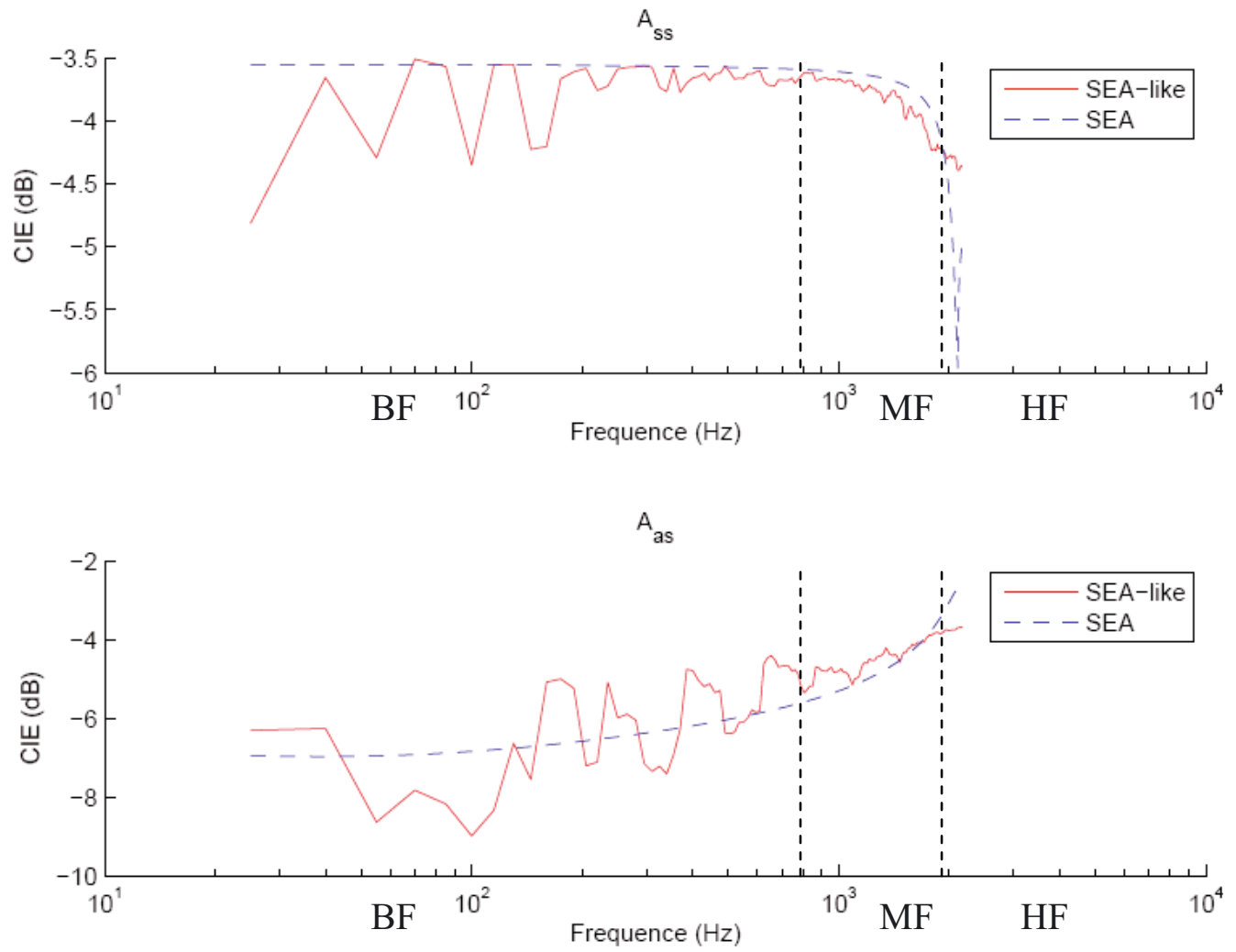

Fig. 2. Comparaison des CIE en SEA-like et SEA. 


\section{Conclusion}

Deux coefficients de la matrice SEA-like du système couplé plaque/cavité ont été calculés puis validés dans le domaine des basses fréquences et des hautes fréquences. Ces validations ont montré que la méthode SEA-like décrit de manière satisfaisante le domaine des moyennes fréquences mais également celui des basses fréquences et celui des hautes fréquences. La méthode de condensation dynamique appliquée au système couplé plaque/cavité permet de simplifier et diminuer les temps de calculs. Il est à noter que la prise en compte des modes découplés de chacun des sous-systèmes entraîne une sous-estimation du couplage, qui peut cependant être négligé et permet la validation des coefficients SEA-like.

Les deux derniers coefficients de la matrice SEA-like sont calculés à partir de l'étude du cas d'excitation de la cavité. Ce cas soulève le problème de la convergence de la pression aux points d'excitation, ce qui rend plus complexe le calcul de la puissance injectée dans la cavité.

Enfin, la méthode SEA-like est appliquée à un cas simple, où les modes sont connus de manière analytique. Un calcul FEM permet de calculer les modes de systèmes plus complexes et d'étendre ainsi la méthode SEA-like à des systèmes industriels présentant une interaction fluide/structure.

\section{Annexe A : Calcul de l'énergie totale dans la plaque}

L'énergie totale de la plaque est la somme de son énergie cinétique et de son énergie de déformation.

L'énergie cinétique de la plaque s'exprime de la façon suivante :

$$
E_{\mathrm{cs}}=\frac{1}{4} h_{\mathrm{s}} \rho_{\mathrm{s}} \int_{A_{\mathrm{s}}} v^{2} \mathrm{~d} s
$$

La vitesse normale dans la plaque est projetée sur les modes découplés :

$$
E_{\mathrm{cs}}=\frac{1}{4} M_{m}^{\mathrm{s}} \sum_{m}\left|V_{m}\right|^{2}
$$

On écrit de même l'énergie de déformation de la plaque :

$$
\begin{array}{r}
E_{p \mathrm{~s}}=\frac{1}{4} D \int_{A_{\mathrm{s}}}\left[|w, x x|^{2}+\mid\right. \\
\\
+2\left(1-\nu_{\mathrm{s}}\right)|w, x y|^{2}+2 \nu_{\mathrm{s}}(w, x x)(w, y y)^{*} \\
\end{array}
$$

ce qui donne :

$$
E_{p \mathrm{~s}}=\frac{1}{16} \frac{D}{\omega^{2}} \pi^{4} A_{\mathrm{s}} \sum_{m}\left|V_{m}\right|^{2}\left(\frac{p_{m}^{2}}{a^{2}}+\frac{q_{m}^{2}}{b^{2}}\right)^{2}
$$

Ainsi l'énergie totale dans la plaque peut s'écrire de la façon suivante :

$$
E_{\mathrm{s}, \mathrm{s}}=\frac{A_{\mathrm{s}}}{16} \sum_{m}\left|V_{m}\right|^{2}\left(\rho_{\mathrm{s}} h_{\mathrm{s}}+\frac{D \pi^{4}}{\omega^{2}}\left(\frac{p_{m}^{2}}{a^{2}}+\frac{q_{m}^{2}}{b^{2}}\right)^{2}\right)
$$

En utilisant les équations (13) et (16), on exprime le vecteur des vitesses modales :

$$
\left|V_{m}\right|^{2}=\frac{1}{4} \frac{\rho_{\mathrm{s}} h_{\mathrm{s}}}{\rho_{0}^{2} c_{0}^{2} A_{\mathrm{s}}} S_{p_{\mathrm{ext}}}(\omega) \sum_{p} \frac{\left|\left(H_{\mathrm{ss}}\right)_{m, p}\right|^{2}}{\left|\chi_{\mathrm{s}, p}\right|^{2}}
$$

où $H_{\mathrm{ss}}$ est la matrice de transfert de la plaque couplée

$$
H_{s s}=\left(\chi_{\mathrm{s}}+{ }^{t} B \chi_{a}^{-1} B\right)^{-1} \chi_{\mathrm{s}}
$$

L'énergie de la plaque est donc donnée par les équations (A.5) et (A.6) :

$$
\begin{aligned}
E_{\mathrm{s}, \mathrm{a}}=\frac{1}{64} \frac{V}{A_{\mathrm{s}}} S_{q_{\mathrm{ext}}} & \sum_{m, p} \frac{\left|\left(H_{\mathrm{sa}}\right)_{m, p}\right|^{2}}{\left|\chi_{\mathrm{a}, p}\right|^{2}} \\
& \times\left(\rho_{\mathrm{s}} h_{\mathrm{s}}+\frac{D \pi^{4}}{\omega^{2}}\left(\frac{p_{m}^{2}}{a^{2}}+\frac{q_{m}^{2}}{b^{2}}\right)^{2}\right)
\end{aligned}
$$

\section{Annexe B : Calcul de l'énergie totale dans la cavité}

L'énergie totale de la cavité est la somme de son énergie cinétique et de son énergie de déformation.

L'énergie potentielle de la cavité s'exprime de la façon suivante :

$$
E_{p \mathrm{a}}=\frac{1}{4 \rho_{0} c_{0}^{2}} \iiint_{V} p p^{*} \mathrm{~d} V
$$

En utilisant l'équation d'Euler, on peut exprimer l'énergie cinétique de la cavité :

$$
E_{c \mathrm{a}}=\frac{1}{4} \frac{1}{\rho_{0} \omega^{2}} \int_{V_{\mathrm{a}}}|\operatorname{grad}(p)|^{2} \mathrm{~d} V
$$

L'énergie totale dans la cavité s'écrit donc :

$$
E_{\mathrm{a}, \mathrm{s}}=\frac{V}{32} \sum_{n}\left|P_{n}\right|^{2}\left(\frac{\pi^{2}}{\rho_{0} \omega^{2}}\left(\frac{m^{2}}{a^{2}}+\frac{n^{2}}{b^{2}}+\frac{t^{2}}{c^{2}}\right)+\frac{1}{\rho_{0} c_{0}^{2}}\right)
$$

De la même manière que précedeméent, on exprime le vecteur des pressions modales dans la cavité :

$$
\left|P_{n}\right|^{2}=\frac{\rho_{0}^{2} c_{0}^{2} V}{8 A_{\mathrm{s}}^{2}} S_{q_{\mathrm{ext}}} \sum_{p} \frac{\left|\left(H_{\mathrm{aa}}\right)_{n, p}\right|^{2}}{\left|\chi_{\mathrm{a}, p}\right|^{2}}
$$

où $H_{\mathrm{aa}}$ est la matrice de transfert de la cavité découplée :

$$
H_{\mathrm{aa}}=\left(I+\chi_{\mathrm{a}}^{-1} B \chi_{\mathrm{s}}^{-1 t} B\right)^{-1}
$$

L'énergie totale dans la cavité s'écrit donc :

$$
\begin{aligned}
E_{\mathrm{s}, \mathrm{a}}=\frac{1}{64} \frac{V}{A_{\mathrm{s}}} S_{q_{\mathrm{ext}}} & \sum_{m, p} \frac{\left|\left(H_{\mathrm{sa}}\right)_{m, p}\right|^{2}}{\left|\chi_{\mathrm{a}, p}\right|^{2}} \\
& \times\left(\rho_{\mathrm{s}} h_{\mathrm{s}}+\frac{D \pi^{4}}{\omega^{2}}\left(\frac{p_{m}^{2}}{a^{2}}+\frac{q_{m}^{2}}{b^{2}}\right)^{2}\right)
\end{aligned}
$$




\section{Références}

[1] B.R. Mace, Statistical energy analysis, energy distribution models and system modes, J. Sound Vib. 264 (2003) 391-409

[2] B.R. Mace, Statistical energy analysis: coupling loss factor, indirect coupling and system modes, J. Sound Vib. 279 (2005) 141-170

[3] B. Hiverniau, Transmissions solidiennes : méthodologie de prévision vibroacoustique moyennes et hautes fréquences sous excitations aéroacoustiques, Thèse, École Centrale de Lyon, 2006

[4] J. Pan, D.A. Bies, The effects of fluid-structural coupling on sound waves in an enclosure, J. Acoust. Soc. Am. 87 (1990) 691-707
[5] K.S. Sum, J. Pan, An analytical model for bandlimited response of acoustical-structural coupled systems. I. Direct sound field excitation, J. Acoust. Soc. Am. 103 (1998) 911-923

[6] K.S. Sum, J. Pan, A study of the medium frequency response of sound field in a panel-cavity system, J. Acoust. Soc. Am. 103 (1998) 1510-1519

[7] R.H. Lyon, R.G. DeJong, Theory and application of statistical energy analysis, Butterworth-Heinemann, Newton, United States, 1995

[8] G. Maidanik, Response of ribbed panels to the reverberant acoustic fields, J. Acoust. Soc. Am. 34 (1962) 809-826 